\title{
Improved Thermal-Vacuum Compatible Flat Plate Radiometric Source For System-Level Testing Of Optical Sensors
}

\author{
Mark A. Schwarz ${ }^{a}$, Craig J. Kent ${ }^{a}$, Robert Bousquet ${ }^{b}$, Steven W. Brown ${ }^{c}$ \\ aStellar Solutions Inc., Palo Alto, CA, USA 94306, 'benesis Engineering Solutions Inc, Lanham, MD, USA 20706, \\ 'National Institute of Standards and Technology, Gaithersburg, MD, USA 20899
}

\begin{abstract}
In this work, we describe an improved thermal-vacuum compatible flat plate radiometric source which has been developed and utilized for the characterization and calibration of remote optical sensors. This source is unique in that it can be used in situ, in both ambient and thermal-vacuum environments, allowing it to follow the sensor throughout its testing cycle. The performance of the original flat plate radiometric source was presented at the 2009 SPIE $^{1}$. Following the original efforts, design upgrades were incorporated into the source to improve both radiometric throughput and uniformity. The pre-thermal-vacuum (pre-TVAC) testing results of a spacecraft-level optical sensor with the improved flat plate illumination source, both in ambient and vacuum environments, are presented. We also briefly discuss potential FPI configuration changes in order to improve its radiometric performance.

Keywords: Calibration, radiometry, remote sensing, source.
\end{abstract}

\section{INTRODUCTION}

Satellite-based sensors typically require sources that can travel with the sensor through all phases of testing, ensuring the quality of its calibration and trending any changes in the sensor that may have occurred postcalibration. The Flat Plate Illuminator (FPI) program developed a vacuum-compatible radiometric source that could be used during the Sensor- and Spacecraft-level testing of the Visible Infrared Imager Radiometer Suite (VIIRS) Sensor as a quality control check for the instrument's radiometric stability. ${ }^{1}$ The first version of the FPI was used during the National Polar orbiting Operational Environmental Satellite System (NPOESS) Preparatory Project (NPP) at the Spacecraft-level for radiometric performance trending of the NPP VIIRS Flight 1 Sensor baseline acceptance testing and Spacecraft thermal-vacuum (TVAC) testing. ${ }^{2}$ After this testing period, areas of improvement in the source were identified and incorporated into an upgraded version of the FPI which was developed and tested. These improvements extended from the NPP FPI ${ }^{1}$ to the next-generation JPSS FPI from 2010 through $2015^{7}$. The improved version of the FPI is currently being utilized for the Joint Polar Satellite System (JPSS), which is the next-generation suite of low earth orbit (LEO) operational environmental satellites. These satellites will circle the Earth approximately once every 100 minutes, providing timely global information about the atmosphere, oceans, land, and near-space environment. ${ }^{1,2}$

\section{GENERAL JPSS FPI DESCRIPTION}

In general, the JPSS FPI consists of five independent, separable components: sources with supporting electronics, a set of optical fiber bundles ${ }^{6}$, the flat plate illuminator itself, the monitor/external detector(s), and, finally, the computer control/software. All of these components have been designed to be transported to a Sensor's test site. The JPSS FPI is equipped with three $300 \mathrm{~W}$ compact $\mathrm{Xe}$ arc sources ${ }^{4}$ and one supercontinuum $^{5}(\mathrm{~S} / \mathrm{C})$ laser-based source from NKT Photonics. 

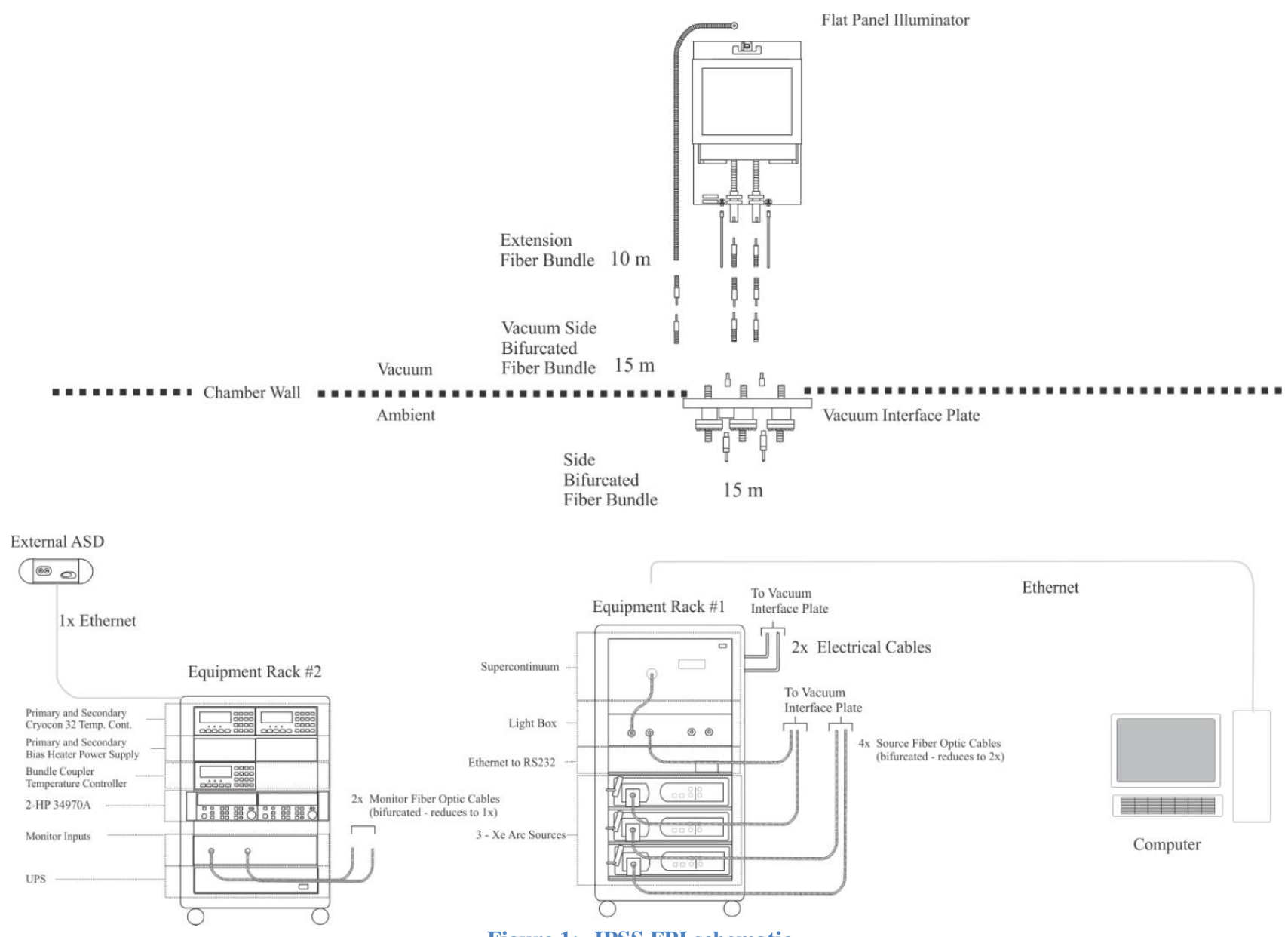

Figure 1: JPSS FPI schematic.

The Xe arc sources consist of the Xe arc lamp, a $3 \mathrm{~mm}$ quartz integrating rod, a variable shutter assembly and a fiber interface/adapter for repeatability when connecting a fiber bundle to the source. The Xe arcs are used as the primary source of radiance for the M1 $(412 \mathrm{~nm})$ and $M 2(445 \mathrm{~nm})$ bands and as a supplement for the radiance in the remaining bands. The shutter sits in between the lamp and the quartz integrating rod and is used to control the amount of source flux coupled into the fiber bundle. The source integrating rods have been decreased from the typical $6 \mathrm{~mm}$ to $3 \mathrm{~mm}$, resulting in a numerical aperture (NA) of approximately 0.5. Each of the three Xe arc sources supplies one of the legs of the bifurcated source fiber bundles. A set of fans has also been added to the Xe arc sources to regulate the temperature of the sourcefiber bundle interface.

A single $S / C$ source is used to provide additional radiance in all of the bands above $M 1$ and $M 2$. The $S / C$ source is operated at a constant output flux and a custom attenuator/coupling box is used to vary the S/C flux into the remaining leg of a bifurcated source fiber bundles. The attenuator box consists of a powered mirror assembly which is used to both reduce the irradiance on a variable (neutral density) attenuator and more efficiently couple the S/C output flux into the FPI fiber bundle.

The shutters and variable attenuator are used in conjunction with the computer control to create a series of radiance levels during the testing. These levels are tuned/measured beforehand using both the external/monitor spectroradiometer, with each source varied to produce a percentage of the band- 
averaged radiance. These levels are reproduced during the Sensor test period for instrument gain trending during the test period. These sources are housed in a rack-mounted shipping container, Rack 1.

A second rack-mount container, Rack 2, holds the heater power supplies, the data acquisition and control hardware, and the communications interfaces (Figure 2). In Rack 2, there are two Cryo-Con temperature controllers (CC1 and CC2) which serve as the primary means for the FPI's temperature control and two Hewlett Packard (HP) 50-W DC heater power supplies (PS1 and PS2), used to provide bias heating when needed. Each of the bias power supplies is capable of maintaining the FPI temperature at $+35^{\circ} \mathrm{C}$ in a liquid nitrogen shroud environment used during testing. An additional temperature controller is used to regulate the temperature of the fiber extension bundle coupler. An Agilent 34970A data acquisition system is used to log the data from both the filter radiometers and thermistors. A monitor spectroradiometer and silicon detector are also housed in Rack 2, providing radiance information while the FPI is being used as a test source. An external spectroradiometer that is maintained under calibration is used to transfer a calibration to the monitor spectroradiometer before and after any sensor testing. All control and data logging communication to the racks/external spectroradiometer is accomplished with a PC computer via Ethernet connection, providing flexibility in the equipment and control computer locations.
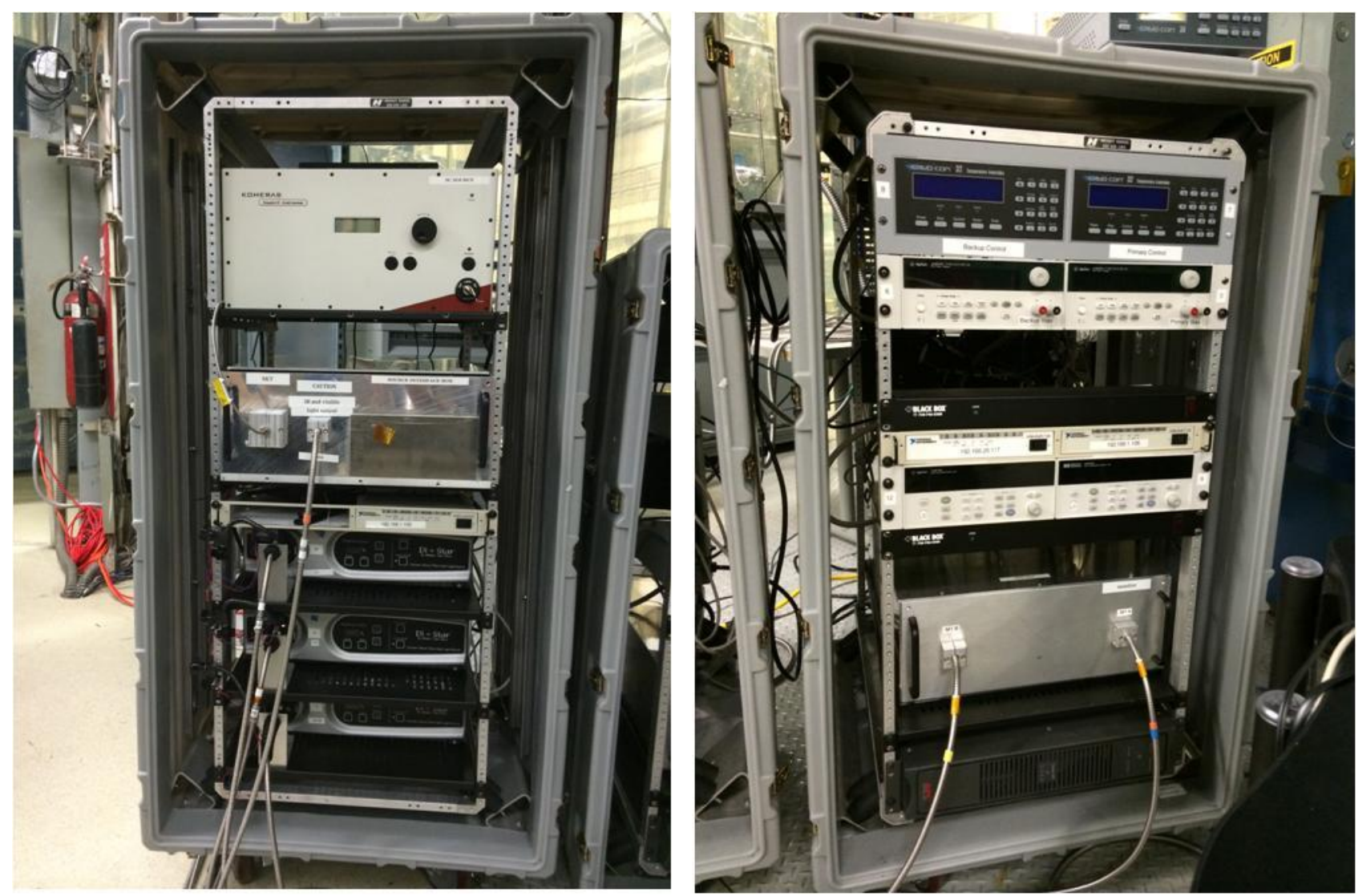

Figure 2. JPSS FPI racks with sources, control electronics, and communications. Rack 1 is on the left; Rack 2 on the right.

A total of six sets of fiber bundles are used with the FPI: three bifurcated $(30 \mathrm{~m})$ and three single fiber bundles $(10 \mathrm{~m})$. The bifurcated fiber bundles contain a vacuum feed-through interface which uses a conflate seal for the vacuum interface. The other half of the fiber flange interfaces sit on a larger o-ring sealed flange that is used as the vacuum interface in the TVAC test vacuum chamber. Two of these (the source bundles) 
are used to transfer the source flux from the Xe arc and S/C sources to the FPI while the remaining (the monitor bundle) has the single ferrule side-mounted above the flat plate. The monitor bundle is used to collect the radiance from the flat plate and couple it to both a spectroradiometer and a silicon detector. The three shorter $10 \mathrm{~m}$ fiber bundles serve as extensions to the bifurcated fiber bundles, facilitating the integration of the FPI into the test vacuum chamber. These fiber bundles directly couple to the bifurcated bundles (i.e. butted end-to-end) via a heated bundle coupler inside the vacuum chamber. The temperature of this coupler is regulated to minimize any thermal effects that might cause radiance variations during TVAC testing.

In addition to the monitor fiber bundle, three Gershun-tube filter radiometers (FRs) monitor the spectral radiance from three of the four corners of the FPI, all of which lie outside of the VIIRS FOV (see Figure 3 ). The filter radiometers are silicon detector elements, one of which is unfiltered. Of the remaining two, one has a $10 \mathrm{~nm}$ Full Width Half Maximum (FWHM) bandpass filter centered at $410 \mathrm{~nm}$, and the other has a 10 $\mathrm{nm}$ FWHM bandpass filter centered at $860 \mathrm{~nm}$. The filter radiometers are located above and to the top and bottom of the flat plate; they view the radiance emitted from the flat plate at approximately $45^{\circ}$ from normal. The silicon detectors are coupled to the FPI wiring harness through BNC-terminated coax cables.

There are two types of electrical harnesses: one interfaces to the heater controllers (heater current and thermistor voltage feedback) and the other collects the filter radiometer signals from the FPI. Both types of harnesses have ambient, vacuum, and extension sections. The extension section (similar to the fiber extensions) facilitates the integration of the FPI into the vacuum chamber. Additionally, a transimpedance amplifier sits on the FPI vacuum flange and relays the detector signal from the extension/vacuum electrical cable to the equipment racks through the ambient electrical cable.

The dimensions of the FPI are approximately $66 \mathrm{~cm}$ by $41 \mathrm{~cm}$ by $14 \mathrm{~cm}$ (not including the standoffs). The FPI radiating surface is approximately $25 \mathrm{~cm}$ by $35 \mathrm{~cm}$. An ambient test fixture has been constructed to allow the FPI to be positioned in a manner similar to the vacuum test fixture which is used during TVAC testing. 


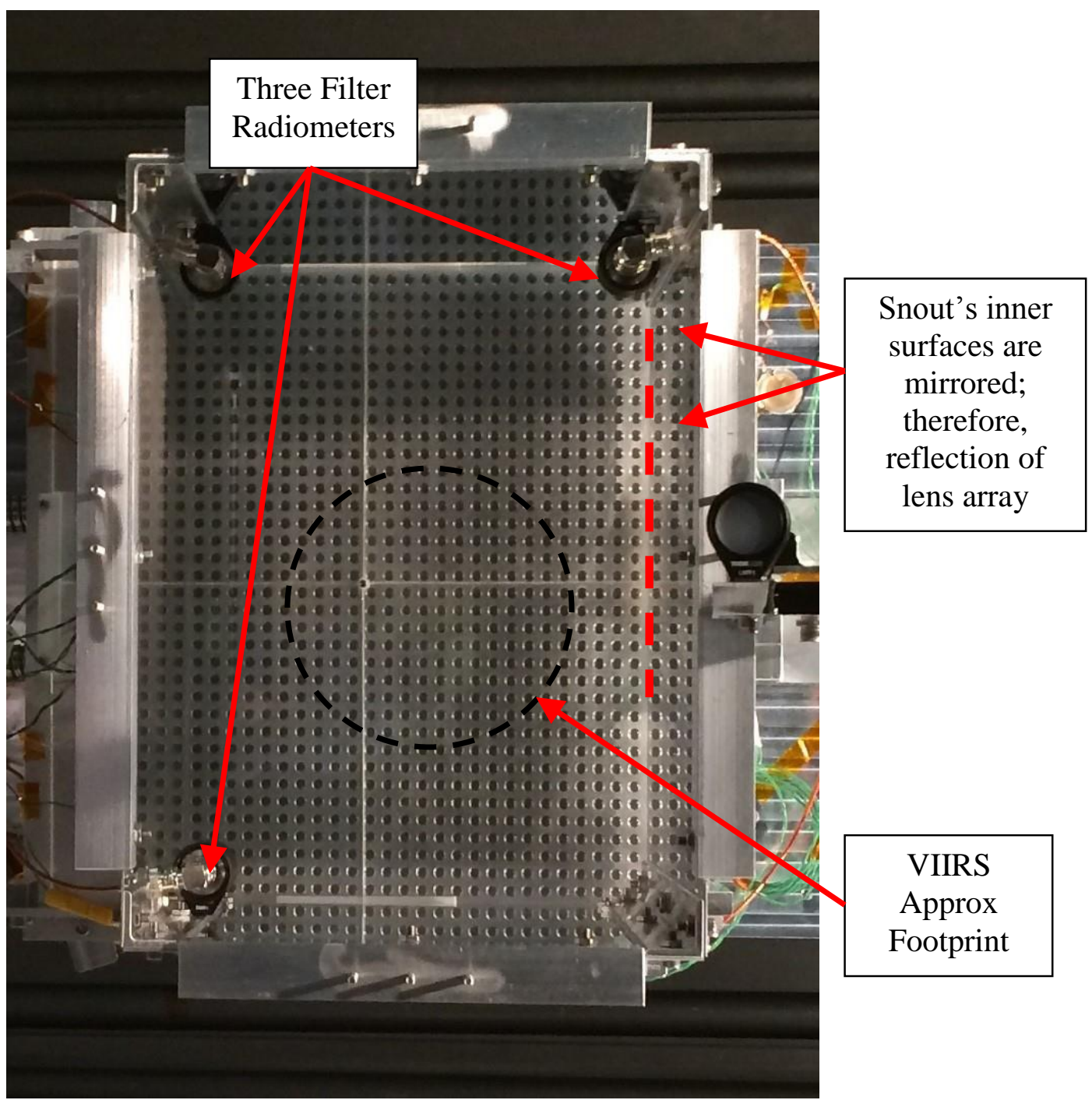

Figure 3. Front view of JPSS FPI showing the three filter radiometers inside the FPI snout. The ground glass diffuser was removed from the front of the snout in order to view the inside.

\section{JPSS FPI RADIOMETRIC PERFORMANCE, PRE-TVAC, IN AMBIENT ENVIRONMENT AT SPACECRAFT-LEVEL}

After the VIIRS Sensor had been integrated onto the Spacecraft mechanical bus, the Spacecraft-level test campaign was carried-out in early 2015 with the FPI to ensure Sensor quality and for continued trending ${ }^{7}$. Following the Spacecraft-level ambient test campaign, the Spacecraft was prepared for TVAC testing, starting in the latter part of 2015, and additional measurements were taken with the FPI, both in ambient and in vacuum (bakeout) environments. These series of measurements were taken with the FPI's radiant sources at $100 \%$ in order to trend the Sensor's performance from the latter part of 2015 through June of 2016. Spacecraft-level TVAC testing is scheduled to start in August 2016. 
Table 1: Comparison of Spacecraft-level Ambient Testing to post-Ambient with FPI sources at $100 \%$.

\begin{tabular}{|l|c|c|c|}
\cline { 2 - 3 } \multicolumn{1}{c|}{} & March 12th 2015 & December 10th 2015 & \multicolumn{1}{|c|}{} \\
\hline Band & $100 \%$ & $100 \%$ & Percent Change \\
\hline M1 & 47.76 & 43.58 & $-8.7 \%$ \\
M3 & 62.26 & 56.89 & $-8.6 \%$ \\
M4 & 96.60 & 91.97 & $-4.8 \%$ \\
\hline M5 & 92.03 & 89.46 & $-2.8 \%$ \\
M6 & 82.68 & 80.06 & $-3.2 \%$ \\
M7 & 68.18 & 66.07 & $-3.1 \%$ \\
M8 & 53.97 & 51.33 & $-4.9 \%$ \\
M9 & 15.09 & 17.16 & $13.7 \%$ \\
M10 & 8.92 & 10.19 & $14.2 \%$ \\
\hline
\end{tabular}

The final set of measurements following the Spacecraft-level ambient test campaign were taken in March of $2015^{7}$. The first set of FPI measurements following the ambient test campaign were taken in December of 2015 , in order to check the health of the FPI. These two sets of band-averaged data, taken with the FPI sources at $100 \%$, are compared in Table 1 , above. There is a noticeable change in the measured irradiance in the blue and NIR bands. The notable change between these two measurements is the configuration for each: The March 2015 data were taken with the fiber bundles partially coiled; the December 2015 data were taken with the fiber bundles coiled in approximately 3 -foot-diameter circles. It is curious that the signal in the blue bands dropped while the signal in the NIR bands increased.

Two subsequent measurements were taken in ambient conditions with the FPI sources at $100 \%$. These were taken in March and May of 2016. Table 2 compares the December 2015 data to the March 2016 data. These data show a much better stability and it was noted that the fiber bundles in both data sets were coiled.

Table 2: Comparison of subsequent ambient measurements taken in Dec 2015 and March 2016 with FPI sources at $100 \%$.

\begin{tabular}{|l|c|c|c|}
\cline { 2 - 4 } \multicolumn{1}{c|}{} & December 10th 2015 & March 30th 2016 & Percent Change \\
\hline Band & $100 \%$ & $100 \%$ & Dec-to-March \\
\hline M1 & 43.58 & 43.33 & $-0.6 \%$ \\
\hline M2 & 56.89 & 56.78 & $-0.2 \%$ \\
M3 & 91.97 & 90.71 & $-1.4 \%$ \\
M4 & 89.46 & 88.68 & $-0.9 \%$ \\
M5 & 80.06 & 78.16 & $-2.4 \%$ \\
M6 & 66.07 & 65.36 & $-1.1 \%$ \\
M7 & 51.33 & 50.10 & $-2.4 \%$ \\
M8 & 17.16 & 17.64 & $2.8 \%$ \\
M9 & 10.19 & 11.21 & $10.0 \%$ \\
M10 & 9.63 & 9.41 & $-2.3 \%$ \\
\hline
\end{tabular}


Yet another set of data were collected in May of 2016 with the FPI's sources at $100 \%$. These data sets are compared in Table 3, below. It was noted that the fiber bundles were coiled during the March 2016 measurements, but were partially coiled (close to final TVAC configuration) during the May 2016 measurements.

Table 3: Comparison of subsequent ambient measurements with FPI sources at $100 \%$.

\begin{tabular}{|l|c|c|c|}
\cline { 2 - 4 } \multicolumn{1}{c|}{} & March 30th 2016 & May 17th 2016 & Percent Change \\
\hline Band & $100 \%$ & $100 \%$ & March-to-May \\
\hline M1 & 43.33 & 42.00 & $-3.1 \%$ \\
M2 & 56.78 & 54.58 & $-3.9 \%$ \\
M3 & 90.71 & 86.48 & $-4.7 \%$ \\
M4 & 88.68 & 83.57 & $-5.8 \%$ \\
M5 & 78.16 & 75.44 & $-3.5 \%$ \\
M6 & 65.36 & 63.23 & $-3.3 \%$ \\
M7 & 50.10 & 49.69 & $-0.8 \%$ \\
M8 & 17.64 & 16.08 & $-8.9 \%$ \\
M9 & 11.21 & 10.09 & $-9.9 \%$ \\
\hline M10 & 9.41 & 8.91 & $-5.3 \%$ \\
\hline
\end{tabular}

\section{JPSS FPI RADIOMETRIC PERFORMANCE, PRE-TVAC, IN VACUUM ENVIRONMENT AT SPACECRAFT-LEVEL}

After the Spacecraft finished its ambient test campaign, the FPI's radiance was measured several times over the course of six months to determine its health. These data were presented in the previous section. The FPI and its associated fiber bundles were then installed in the TVAC chamber in order to vacuum-bakeout the hardware, preparing for the Spacecraft-level TVAC test campaign (scheduled for the latter part of 2016). Following the first vacuum-bakeout cycle, the chamber was set to a cold-plateau soak. The FPI's sources were turned on and set to 100\% during this soak phase. Table 4 compares the ambient data taken in May 2016 to the vacuum data taken in June 2016. In both cases, the fiber bundles were in their final TVAC configuration, being partially coiled. 
Table 4: Comparison of May 2016 ambient measurement to June 2016 vacuum with FPI sources at $100 \%$.

\begin{tabular}{|l|c|c|c|}
\cline { 2 - 3 } \multicolumn{1}{c|}{} & May 17th 2016 & June 4th 2016 & \multicolumn{1}{|c|}{} \\
\hline Band & 100\%, ambient & 100\%, vacuum & Percent Change \\
\hline M1 & 42.00 & 41.67 & $-0.8 \%$ \\
\hline M2 & 54.58 & 53.17 & $-2.6 \%$ \\
M3 & 86.48 & 84.08 & $-2.8 \%$ \\
M4 & 83.57 & 81.04 & $-3.0 \%$ \\
\hline M5 & 75.44 & 72.68 & $-3.7 \%$ \\
\hline M6 & 63.23 & 59.26 & $-6.3 \%$ \\
\hline M8 & 49.69 & 46.50 & $-6.4 \%$ \\
M9 & 16.08 & 14.70 & $-8.6 \%$ \\
M10 & 10.09 & 8.91 & $-11.8 \%$ \\
\hline
\end{tabular}

The longer-wavelength bands exhibit a noticeable drop in their band-averaged signal. This disparity is currently being investigated, possibly related to the ASD gain settings or with the supercontinuum laser source that produces the energy for those wavebands.

\section{SUMMARY AND CONCLUSIONS}

In this work, we have described the results from pre-TVAC Spacecraft-level testing, in both ambient and vacuum environments, with a new class of vacuum-compatible, externally illuminated, fiber-optically coupled, flat plate illuminator for the radiometric characterization and calibration of large aperture sensors. There are many challenges associated with providing a uniform radiance source that can be used in both ambient and vacuum conditions, and at the same time, is transportable to the test sites. The FPI provides a novel means of accomplishing this versus the alternative (i.e. reflectance plaques, integrating spheres, etc).

The JPSS FPI represented in this work has an active area of $25 \mathrm{~cm}$ by $35 \mathrm{~cm}$ and a total depth under $20 \mathrm{~cm}$. The overall footprint is less than $1 \mathrm{~m}^{2}$. The compact size and fiber bundles allow this type of source to be placed and operated in confined spaces, for example, in a vacuum chamber, over a wide range of environmental temperatures. Other potential applications include, but are not limited to, providing a calibration of aircraft sensors in situ in the aircraft instrument bay just prior to and following a flight; also, configurations for in-flight calibration of aircraft sensors can also be envisioned. There is no limitation on the size of these sources or on the number of optical fibers terminated in the source plane. Consequently, applications related to the near-field characterization of larger aperture sensors, e.g. astronomical telescopes with meter-size primary mirrors, may also be possible.

\section{ACKNOWLEDGEMENTS}

We would like to acknowledge and thank NIST colleague, Dr John Woodward, for his assistance in the functional checkout of the JPSS FPI. 
* Identification of commercial equipment does not imply recommendation or endorsement by Stellar Solutions or the National Institute of Standard and Technology, nor does it imply that the equipment identified is necessarily the best available for the purpose.

\section{REFERENCES}

1. S.W. Brown, et al, "A vacuum-compatible flat plate radiometric source for system-level testing of optical sensors," Proc. SPIE 7474, Sensors, Systems, and Next-Generation Satellites XIII, 747412 (September 24, 2009)

2. R.R. Bousquet, et al, "Spacecraft level radiometric testing with the flat plate illuminator," Cal Con Technical Conference (2010)

3. RPC Photonics, http://www.rpcphotonics.com

4. Perkin Elmer XL3000 compact Xe arc source, http://optoelectronics.perkinelmer.com

5. NKT Photonics, http://www.nktphotonics.com

6. CeramOptec, http://www.ceramoptec.com

7. M.A. Schwarz, et al, "Improved Thermal-Vacuum Compatible Flat Plate Radiometric Source For System-Level Testing Of Optical Sensors," Proc. SPIE 9607-34, Earth Observing Systems XX, (August 11, 2015) 